Pacific Journal of Mathematics

MINIMAL NONPERMUTATIVE PSEUDOVARIETIES OF 


\title{
MINIMAL NON-PERMUTATIVE PSEUDOVARIETIES OF SEMIGROUPS. I
}

\author{
JORGE AlMEIDA
}

\begin{abstract}
A semigroup is permutative if it satisfies an identity of the form $x_{1} x_{2} \cdots x_{n}=x_{\sigma 1} x_{\sigma 2} \cdots x_{\sigma n}$ where $\sigma$ is a non-identical permutation of $\{1,2, \ldots, n\}$. The finite permutative semigroups form a pseudovariety and permutative pseudovarieties enjoy many properties first obtained for commutative pseudovarieties. Several types of permutation identities are considered, and all pseudovarieties minimal with respect to the property of containing a finite semigroup which fails an identity of a given type are determined. This includes the cases of the commutativity identity, the general permutation identities, and the "strong (left) permutation identities". As a preliminary, all minimal non-commutative pseudovarieties of groups and monoids are also determined.
\end{abstract}

1. Introduction. As a natural generalization of commutativity, permutation identities have been considered by several authors and shown to play an important role in various contexts (see Yamada [19, 20], Perkins [12], Pollák [14, 15], Higgins [7, 8], Almeida [1]). The non-collapse of this extended concept is peculiar to the class of semigroups, as a permutative monoid is necessarily commutative.

The problem of determining the minimal non-commutative pseudovarieties of groups was first considered by S. Oates (see Neumann [11], p. 42) although this author did not exhibit all such pseudovarieties, rather just established that they must contain a non-abelian metabelian group. As a step towards the solution of a problem in language theory, Margolis and Pin [10] then extended this result by showing that a non-commutative pseudovariety of monoids all of whose group members are abelian must contain one of three monoids which they describe. They also claim that their methods can be adjusted to yield a list of generators (up to the group case) of the minimal non-commutative pseudovarieties of semigroups. However, as we show here, their list is incomplete.

In the first part of this work, we determine explicitly all minimal non-commutative pseudovarieties of groups and semigroups. We also consider some special types of permutation identities and produce a complete list of minimal non-strongly permutative and non-strongly left permutative pseudovarieties of semigroups. This is based on the solution 
of the monoid case, together with the reduction of the statement that a semigroup $S$ satisfies a certain kind of permutation identity to a much simpler statement of pseudo-identical type involving idempotents (cf. Reiterman [18]). In this way, we eliminate the bothersome presence of existential quantifiers on natural numbers.

All minimal pseudovarieties with respect to failing one of the above properties are characterized here both by a generator of minimum size and by a finite basis of identities.

Part II of this work presents a complete list of minimal non-permutative pseudovarieties of semigroups (with no restriction on the nature of the excluded permutation identities).

The author is indebted to Professor Norman Reilly for his encouragement and many helpful discussions during the preparation of this paper.

2. Preliminaries. For general undefined terms and background, we refer the reader to Clifford and Preston [3] and Lallement [9]. For the notion of "pseudovariety" and its relationship with varieties in Birkhoff's sense, see Ash [2]. Finally, the definition of "implicit operation" on a class of algebras and its connection with pseudovarieties can be found in Reiterman [18].

Here, we will call pseudo-identity an expression of the form $\pi=\rho$, where $\pi$ and $\rho$ are implicit operations on the class of all finite semigroups. For a set $\Sigma$ of pseudo-identities, $\llbracket \Sigma \rrbracket$ denotes the class of all finite semigroups satisfying $\Sigma$. By Theorem 3.1 [18], every pseudovariety is of the form $\llbracket \Sigma \rrbracket$ for some set $\Sigma$ of pseudo-identities, while by Propositions 1.2 and 2.1 [18], every pseudovariety which is generated by a single semigroup is of the form $\llbracket \Sigma \rrbracket$ for some set $\Sigma$ of identities.

For a finite semigroup $S, \underline{V}(S)$ denotes the pseudovariety generated by $S$. Thus $\underline{V}(S)$ consists of all homomorphic images of finite subdirect powers of $S$. As usual, $S^{1}$ stands for the smallest monoid containing $S$, i.e., $S^{1}=S$ if $S$ is a monoid, otherwise $S^{1}=S \cup\{1\}$ where 1 acts as an identity. Next, $S^{r}$ denotes the set $S$ under the reverse operation $a * b=b a$, and $E(S)$ represents the set of all idempotents in $S$. If $A, B \subseteq S$, we let $A B=\{a b: a \in A, b \in B\}$.

For a set $P$, we let $|P|$ denote its cardinality.

Our first result is a simple sharpening of Proposition III. 9.2 of Eilenberg [6] and will be of crucial importance later.

Proposition 2.1. Let $S$ be a finite semigroup and $E=E(S)$. Let $n=|S|, k=|E|$. Then, either $S^{n-k}=S E S$ or $E$ is a band ideal of $S$ with $S / E$ cyclic (and so $S^{n-k+1}=E$ ). In particular, $S^{n-k+1}=S E S$. 
Proof. We first show that $S^{n-k+1}=S E S$. Let $s_{1}, s_{2}, \ldots, s_{n-k+1} \in S$ and let $t_{i}=s_{1} s_{2} \cdots s_{i}$. If the elements $t_{i}(i=1, \ldots, n-k+1)$ are all distinct, then $t_{i}=e \in E$ for some $i$ and so $s_{1} s_{2} \cdots s_{n-k+1}=s_{1} \cdots s_{i} \cdot e$ $\cdot e s_{i+1} \cdots s_{n-k+1} \in S E S$. If $t_{i}=t_{j}$ with $i<j$, let $\left(s_{i+1} s_{i+2} \cdots s_{j}\right)^{l}=$ $e \in E$, to obtain $t_{i}=t_{i} e$ and so $s_{1} s_{2} \cdots s_{n-k+1}=s_{1} \cdots s_{i} \cdot e \cdot s_{i+1} \cdots$ $s_{n-k+1} \in S E S$. Hence $S^{n-k+1} \subseteq S E S$. Since $S E S \subseteq S^{r}$ for all $r \geq 1$, we conclude that $S^{n-k+1}=S E S$.

Next, suppose $S \neq E$, i.e., $k<n$, and further suppose that $S^{n-k} \neq$ $S E S$. Let $s_{1}, s_{2}, \ldots, s_{n-k} \in S$ be such that $s_{1} s_{2} \cdots s_{n-k} \notin S E S$. By the same argument as above, it follows that the elements $t_{i}=s_{1} s_{2} \cdots s_{i}$ $(i=1, \ldots, n-k)$ are all distinct and non-idempotent. Thus, $S=$ $\left\{t_{1}, t_{2}, \ldots, t_{n-k}\right\} \cup E$ and $E$ is an ideal of $S$. In particular, $E$ is a band.

With the previous choice of elements $s_{1}, s_{2}, \ldots, s_{n-k} \in S$, if $s_{i} \neq s_{1}$ for some $i$, then, since $s_{i} \notin E$, we must have $s_{i}=t_{j}$ for some $j \in$ $\{2, \ldots, n-k\}$. Hence, $s_{1} s_{2} \cdots s_{n-k} \in S^{n-k+1}=S E S$, a contradiction. Therefore, $s_{1}=s_{2}=\cdots=s_{n-k}$ and so $s_{1}$ generates the Rees quotient $S / E$.

A permutation identity is an identity of the form

$$
x_{1} x_{2} \cdots x_{n}=x_{\sigma 1} x_{\sigma 2} \cdots x_{\sigma n}
$$

where $\sigma$ is a permutation of $\{1,2, \ldots, n\}$ and $x_{1}, x_{2}, \ldots, x_{n}$ are distinct variables. We say that a semigroup $S$ is permutative if it satisfies some nontrivial (i.e., $\sigma \neq \imath$ ) permutation identity. $S$ is strongly left (resp. right) permutative if it satisfies an identity (1) with $\sigma 1 \neq 1$ (resp. $\sigma n \neq n$ ). $S$ is strongly permutative if it is both strongly left and right permutative. Note that a permutative monoid is commutative.

We denote by Perm $_{(k, m, l)}$ the pseudovariety defined by the identities of the form (1) with $n=k+m+l$ and $\sigma i=i$ for $1 \leq i \leq k$ and for $n-l+1 \leq i \leq n$, where $k, l \geq 0$ and $m \geq 2$. Then, $\underline{P e r m}_{(0,2,0)}=$ Com is the class of all finite commutative semigroups, and we let

$$
\begin{aligned}
& \underline{\operatorname{Perm}}_{(0, \infty, 0)}=\bigcup_{m \geq 2} \underline{\operatorname{Perm}}_{(0, m, 0)}, \\
& \underline{\text { Perm }}_{(0, \infty, \infty)}=\bigcup_{m \geq 2, l \geq 0} \underline{\text { Perm }}_{(0, m, l)}, \text { and } \\
& \underline{\text { Perm }}=\bigcup_{k, l \geq 0, m \geq 2} \underline{\text { Perm }}_{(k, m, l)} .
\end{aligned}
$$

These classes of finite semigroups are all pseudovarieties and are identified by other means in the next section. 
3. Simplification of permutativity conditions using idempotents. In this section, we take advantage of the abundance of idempotents in finite semigroups as expressed in Proposition 2.1 to obtain equivalent conditions to various types of permutativity.

LEMMA 3.1. Let $S$ be a strongly left permutative semigroup. Then, for any $e \in E(S), s \in e S e$ and $t \in S$, we have $t s=$ ste.

Proof. Let $S$ satisfy the identity (1) with $\sigma 1 \neq 1$. Let $\alpha=\sigma^{-1}(1)$ and let $r, s, t$ be as in the statement of the lemma. Then

$$
t s=t e^{\alpha-2} s e^{n-\alpha}=s e^{*} t e^{*}=\text { ste. }
$$

LEMMA 3.2. Let $S$ be a finite semigroup with $n$ elements and let $E=E(S)$. Suppose that all submonoids of $S$ are commutative.

(i) If, for any $e \in E$ and $t \in S$, te $=$ ete, then $S \in \underline{\operatorname{Perm}}_{(0,2, n-1)}$.

(ii) If every idempotent in $S$ is central, then $S \in \underline{\text { Perm }}_{(0, m, 0)}$ where $m=\max \{2, n-1\}$.

Proof. By Proposition 2.1, we have either $S^{n-1}=S E S$, or $|E|=1$ and $S$ is commutative. Thus, we may assume that $S^{n-1}=S E S$.

(i) Since $S E=E S E \subseteq E S$, we deduce $S^{n-1}=E S$. Let $s_{1}, s_{2}$, $t_{1}, \ldots, t_{n-1} \in S$. Then $t_{1} t_{2} \cdots t_{n-1}=e t^{\prime}$ for some $e \in E$ and $t^{\prime} \in S$. Hence, $t_{1} t_{2} \cdots t_{n-1}=e t_{1} t_{2} \cdots t_{n-1}$, and so

$$
\begin{aligned}
s_{1} s_{2} t_{1} \cdots t_{n-1} & =s_{1} s_{2} e t_{1} \cdots t_{n-1} \\
& =e s_{1} e s_{2} e t_{1} \cdots t_{n-1} \quad \text { since } t e=e t e \\
& =e s_{2} e s_{1} e t_{1} \cdots t_{n-1} \quad \text { since } e S e \text { is commutative } \\
& =s_{2} s_{1} e t_{1} \cdots t_{n-1}=s_{2} s_{1} t_{1} \cdots t_{n-1} .
\end{aligned}
$$

This establishes $S \in \underline{\operatorname{Perm}}_{(0,2, n-1)}$.

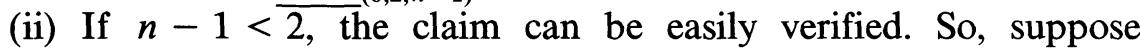
$m=n-1$ and let $s_{1}, s_{2}, \ldots, s_{m} \in S$. Since $S^{n-1}=S E S$ and idempotents are central, for each permutation $\sigma$ of $\{1,2, \ldots, m\}$, there exists $e_{\sigma} \in E$ such that $s_{\sigma 1} s_{\sigma 2} \cdots s_{\sigma m}=e_{\sigma} s_{\sigma 1} s_{\sigma 2} \cdots s_{\sigma m}$. Then, for every such $\sigma$, we have

$$
\begin{aligned}
s_{\sigma 1} s_{\sigma 2} \cdots & s_{\sigma m}=\left(e_{\sigma} s_{\sigma 1}\right)\left(e_{\sigma} s_{\sigma 2}\right) \cdots\left(e_{\sigma} s_{\sigma m}\right) \quad \text { since } e_{\sigma} \text { is central } \\
& =\left(e_{\sigma} s_{1}\right)\left(e_{\sigma} s_{2}\right) \cdots\left(e_{\sigma} s_{m}\right) \quad \text { since } e_{\sigma} S=e_{\sigma} S e_{\sigma} \text { is commutative } \\
& =e_{\sigma} s_{1} s_{2} \cdots s_{m},
\end{aligned}
$$


so that, if $\iota$ denotes the identity permutation of $\{1,2, \ldots, m\}$, then

$$
\begin{aligned}
s_{\sigma 1} s_{\sigma 2} \cdots s_{\sigma m} & =e_{\sigma} s_{1} \cdots s_{m}=e_{\sigma} e_{\iota} s_{1} \cdots s_{m}=e_{\iota} e_{\sigma} s_{1} \cdots s_{m} \\
& =e_{\iota} s_{\sigma 1} \cdots s_{\sigma m}=e_{\iota} s_{1} \cdots s_{m}=s_{1} \cdots s_{m},
\end{aligned}
$$

as desired.

THEOREM 3.3. The following conditions are equivalent for a finite semigroup $S$.

(i) $S \in \operatorname{Perm}_{(0, \infty, 0)}$.

(ii) $S \in \overline{\operatorname{Perm}}_{(0, m, 0)}$ where $m=\max \{2,|S|-1\}$.

(iii) $S$ is strongly permutative.

(iv) All submonoids of $S$ are commutative and every idempotent of $S$ is central.

(v) Every element of $S$ which lies in some submonoid of $S$ is central in $S$.

Proof. (ii) $\Rightarrow$ (i) $\Rightarrow$ (iii) follow directly from the definitions, while (v) $\Rightarrow$ (iv) is obvious. (iii) $\Rightarrow$ (v) follows from Lemma 3.1 and its left-right dual. Finally, (iv) $\Rightarrow$ (ii) is Lemma 3.2(ii).

Notation. $x^{\omega}$ denotes the implicit unary operation on finite semigroups which associates with an element $s$ of a finite semigroup $S$ the unique idempotent in the subsemigroup of $S$ generated by $s$.

COROllary 3.4. $\underline{\operatorname{Perm}}_{(0, \infty, 0)}=\llbracket x^{\omega} y z=z y x^{\omega} \rrbracket$.

THEOREM 3.5. The following conditions are equivalent for a finite semigroup $S$.

(i) $S \in \operatorname{Perm}_{(0, \infty, \infty)}$.

(ii) $S \in \overline{\operatorname{Perm}}_{(0,2, n-1)}$ where $n=|S|$.

(iii) $S$ is strongly left permutative.

(iv) All submonoids of $S$ are commutative and, for any $e \in E(S)$ and $t \in S$, te $=$ ete.

(v) For any $e \in E(S)$ and $s, t \in S$, este $=t s e$.

Proof. The proof proceeds along the same lines as the proof of Theorem 3.3 with the difference that in establishing (iii) $\Rightarrow(v)$, one first uses Lemma 3.1 to obtain te $=$ ete and then deduces that

$$
\text { este }=\text { esete }=\text { etese }=\text { tse. }
$$

Corollary 3.6. $\underline{\operatorname{Perm}}_{(0, \infty, \infty)}=\llbracket x^{\omega} y z x^{\omega}=z y x^{\omega} \rrbracket$. 
LEMMA 3.7. Let $S$ be a permutative semigroup. Then, for any $e$, $f \in E(S)$ and $s, t \in S$, we have est $f=$ etsf.

Proof. Let $S$ satisfy the identity (1) with $k+1=\min \{i: \sigma i \neq i\}$ and $n-l=\max \{j: \sigma j \neq j\}$. Then

$$
\begin{aligned}
\text { estf }=e^{k-1} s t f^{n-k-1} & =e^{k-1} s f f^{*} t f^{*} f^{l} \quad \text { using (1) } \\
& =\text { esftf. }
\end{aligned}
$$

Hence,

$$
\begin{aligned}
\text { estf }=\text { esftf } & =(e \cdot e s \cdot f) t f \\
& =e \cdot e f s \cdot f t f \quad \text { by the above argument } \\
& =e f t f s f \text { since } f S f \text { is commutative } \\
& =e t s f \quad \text { by the above. }
\end{aligned}
$$

LeMma 3.8. Let $S$ be a finite semigroup with $n$ elements. Suppose that for any $e, f \in E=E(S)$ and $s, t \in S$, we have estf = etsf. Then $S$ $\in \underline{\operatorname{Perm}}_{(n-1,2, n-1)}$.

Proof. As in the proof of Lemma 3.2, we may assume that $S^{n-1}=$ $S E S$. Let $m=n-1$ and let $s_{1}, \ldots, s_{m}, t_{1}, t_{2}, u_{1}, \ldots, u_{m} \in S$. Then, there exist $e, f \in E$ and $s^{\prime}, s^{\prime \prime}, u^{\prime}, u^{\prime \prime} \in S$ such that $s_{1} \cdots s_{m}=s^{\prime} e s^{\prime \prime}$ and $u_{1} \cdots u_{m}=u^{\prime} f u^{\prime \prime}$. Hence,

$$
\begin{aligned}
s_{1} \cdots s_{m} t_{1} t_{2} u_{1} \cdots u_{m} & =s^{\prime} e s^{\prime \prime} t_{1} t_{2} u^{\prime} f u^{\prime \prime} \\
& =s^{\prime} e s^{\prime \prime} t_{2} t_{1} u^{\prime} f u^{\prime \prime} \quad \text { since } \text { estf }=\text { etsf } \\
& =s_{1} \cdots s_{m} t_{2} t_{1} u_{1} \cdots u_{m},
\end{aligned}
$$

as desired.

THEOREM 3.9. The following are equivalent for a finite semigroup $S$.

(i) $S \in$ Perm.

(ii) $S \in \overline{\text { Perm }}_{(n-1,2, n-1)}$ where $n=|S|$.

(iii) $S$ is permutative.

(iv) All submonoids of $S$ are commutative and, for any $e, f \in E(S)$ and $s, t \in S$, esf $=$ esef and este $=$ esete.

(v) For any e, $f \in E(S)$ and $s, t \in S$, estf $=$ etsf.

Proof. Here, it is now sufficient to check that (iv) $\Leftrightarrow$ (v). (v) $\Rightarrow$ (iv) is easily verified. Conversely, assume (iv) and let $e, f \in E(S)$ and $s, t \in S$. Then,

$$
\text { est } f=\text { estef }=\text { esetef }=\text { etesef }=e t s f,
$$

as claimed. 
COROllary 3.10.

Perm $=\llbracket x^{\omega} y z t^{\omega}=x^{\omega} z y t^{\omega} \rrbracket=\llbracket x^{\omega} y z x^{\omega}=x^{\omega} z y x^{\omega}, x^{\omega} y z^{\omega}=x^{\omega} y x^{\omega} z^{\omega} \rrbracket$.

EXAMPLE 3.11. Let $D$ be the semigroup with zero with presentation

$$
\left\langle e, f ; e=e^{2}, f=f^{2}, f e=0\right\rangle .
$$

Then $D=\{0, e, f, e f\}$ satisfies $x y z x=x z y x$ and so monoids in $\underline{V}(D)$ are commutative. However, $e \cdot e f \cdot f=e f \neq 0=e \cdot f e \cdot f$, and so $D$ is nonpermutative by Theorem 3.9(v).

REMARK 3.12. If an arbitrary semigroup $S$ satisfies an identity $\varepsilon$ of the form (1) with $L(\varepsilon)=\min \{i: \sigma i \neq i\}$ and $R(\varepsilon)=n_{\varepsilon}-\max \{j: \sigma j \neq j\}$, then $S$ satisfies all the identities $\delta$ of the form (1) with $L(\delta) \geq L(\varepsilon)$ and $R(\delta) \geq R(\varepsilon)$ for which the length $n_{\delta}$ is large enough (independently of $S$ ). This follows from the results of Putcha and Yaqub [16] (see also Pollák [13]).

REMARK 3.13. The bound $n-1$ in parts (ii) of Theorems 3.3, 3.5 and 3.9 is sharp. For instance, if $S$ is a two-element left-zero semigroup, then $S \in \underline{\text { Perm }}_{(1,2,1)} \backslash \underline{\text { Perm }}_{(0,2,0)}$.

4. Minimal non-commutative pseudovarieties of groups. Since a finite semigroup is a group if and only if it satisfies identities of the form $x y^{n}=x=y^{n} x$ for some $n$, every pseudovariety of groups can be viewed as a pseudovariety of semigroups. Moreover, as a pseudovariety which is generated by a single semigroup is defined by identities, it follows that the pseudovarieties indicated in the title of this section are essentially the same whether we work in the algebraic types of groups or semigroups. To simplify the notation, we will consider groups (rather than semigroups which are groups), thus referring to the unary operation of inversion.

A group is said to be one-step non-commutative if it is non-abelian but all its proper subgroups are abelian. We proceed to describe all such finite groups. In the following, $p$ and $q$ always denote prime numbers.

THEOREM 4.1. (Rédei [17].) (i) The finite one-step non-commutative p-groups are the quaternion group (or order 8), the groups (of order $p^{m+n+1}$ ) defined by the relations

$$
a^{p^{m}}=b^{p^{n}}=1, \quad c^{p}=1, \quad a c=c a, \quad b c=c b, b a b^{-1}=a c
$$


and the group (of order $p^{m+n}$ ) defined by the relations

$$
a^{p^{m}}=1, \quad b^{p^{m}}=1, \quad b a b^{-1}=a^{1+p^{m-1}} \quad(m \geq 2, n \geq 1) .
$$

(ii) The remaining finite one-step non-commutative groups are obtained as follows. For two distinct primes $p, q$ and natural number $n$, let $m$ denote the multiplicative order of $p$ modulo $q$ and let $F=\mathrm{GF}\left(p^{m}\right)$. Choose $\omega \in F^{*}$ of order $q$. Take for the group the semidirect product $F * \mathbf{Z}_{q^{n}}$ with product given by

$$
(\alpha, a)(\beta, b)=\left(\alpha+\omega^{a} \beta, a+b\right) \quad\left(\alpha, \beta \in F, a, b \in \mathbf{Z}_{q^{n}}\right) .
$$

LEMMA 4.2. (i) If $G$ and $G^{\prime}$ are two p-groups defined by (2) with parameters $m, n$ and $m^{\prime}, n^{\prime}$ respectively with $m \leq m^{\prime}$ and $n \leq n^{\prime}$, then $G$ is a homomorphic image of $G^{\prime}$.

(ii) If $H$ and $H^{\prime}$ are two groups defined by (4) relative to the same pair of primes, with parameters $n$ and $n^{\prime}$ respectively and $n \leq n^{\prime}$, then $H$ is a homomorphic image of $H^{\prime}$.

Proof. (i) The subgroup $K$ of $G^{\prime}$ generated by $\left\{a^{p^{m^{\prime}-m}}, b^{p^{n^{\prime}-n}}\right\}$ is central and $G^{\prime} / K \simeq G$.

(ii) The subgroup of $H^{\prime}$ generated by $\left(0, q^{n^{\prime}-n}\right)$ is central and $H^{\prime} / K$ $\simeq H$.

We let $[x, y]=x^{-1} y^{-1} x y,\left[x_{1}, x_{2}, \ldots, x_{n+1}\right]=\left[\left[x_{1}, \ldots, x_{n}\right], x_{n+1}\right]$, $\left[x,(y)_{1}\right]=[x, y]$, and $\left[x,(y)_{n+1}\right]=\left[\left[x,(y)_{n}\right], y\right]$. The following commutator identities are easily verified (in any group):

$$
\begin{aligned}
{[x, y] } & =[y, x]^{-1} \\
x y & =y x[x, y] \\
{[x, y z] } & =[x, z] z^{-1}[x, y] z=[x, z][x, y][x, y, z] .
\end{aligned}
$$

We denote the group given by (2) with $m=n=1$ by $G_{p}$, while $H_{p, q}$ represents the group given by (4) when $n=1$.

LEMMA 4.3. (i) The pseudovariety generated by any group $K$ in (3) is given by

$$
\underline{V}(K)=\llbracket x^{p^{s}}=1,[x, y]^{p}=1,[x, y, z]=1 \rrbracket
$$

$$
\text { where } s=\max \{m, n\} \text {. }
$$

(ii) The pseudovariety generated by the group $G_{p}$ is given by

$$
\begin{gathered}
\underline{V}\left(G_{p}\right)=\llbracket x^{p}=1,[x, y, z]=1 \rrbracket \quad \text { in case } p>2, \\
\underline{V}\left(G_{2}\right)=\llbracket x^{4}=1,[x, y]^{2}=1,[x, y, z]=1 \rrbracket .
\end{gathered}
$$


Proof. It is easily checked that $K$ satisfies the identities in (5). Using those identities, any word $w$ in the variables $x_{1}, x_{2}, \ldots, x_{n}$ can be reduced to one of the form $x_{1}^{u_{1}} \cdots x_{r}{ }^{u_{n}} c_{1}{ }^{v_{1}} c_{2}{ }^{v_{2}} \cdots c_{t}^{v_{t}}$ where the $c_{k}$ are distinct commutators of the form $\left[x_{i}, x_{j}\right]$ with $i<j$. Then, one checks that $K$ satisfies $w=1$ if and only if $p^{s} \mid u_{i}(i=1, \ldots, r)$ and $p \mid v_{j}(j=1, \ldots, t)$. This establishes (i). The proof of (ii) is similar.

Proposition 4.4.

$$
\begin{aligned}
& \underline{V}\left(H_{p, q}\right)=\llbracket x^{p q}=1,\left[x^{q}, y^{q}\right]=[x, y]^{p}=1, \\
& {[y, x, z][x, y, z]=1,[x, y, z, t]=[x, y, t, z],} \\
& {[x, y]^{q}\left[x,(y)_{2}\right]^{\left(\frac{q}{2}\right)} \cdots\left[x,(y)_{q-1}\right]^{\left(q^{q}-1\right)}\left[x,(y)_{q}\right]=1 \rrbracket .}
\end{aligned}
$$

Proof. We first observe that $(\alpha, a)^{-1}=\left(-\omega^{-a} \alpha,-a\right)$ while, for $r \geq 1$,

$$
(\alpha, a)^{r}=\left(\left(1+\omega^{a}+\omega^{2 a}+\cdots+\omega^{(r-1) a}\right) \alpha, r a\right) .
$$

Further, if $u=(\alpha, a), v=(\beta, b)$, then

$$
[u, v]=\left(\left(\omega^{-b}-1\right) \omega^{-a} \alpha-\left(\omega^{-a}-1\right) \omega^{-b} \beta, 0\right) ;
$$

in particular, $[(\alpha, 0),(\beta, b)]=\left(\left(\omega^{-b}-1\right) \alpha, 0\right)$. Also, note that, in $F[X]$,

$$
(X+1)^{q}-1=q X+\left(\begin{array}{l}
q \\
2
\end{array}\right) X^{2}+\left(\begin{array}{l}
q \\
3
\end{array}\right) X^{3}+\cdots+\left(\begin{array}{c}
q \\
q-1
\end{array}\right) X^{q-1}+X^{q}
$$

admits the factorization $\prod_{a=0}^{q}\left(X-\omega^{a}+1\right)$. Using these remarks, it is easy to check that $H_{p, q}$ satisfies the identities in (8).

Now, let $w$ be a word in the variables $x_{1}, x_{2}, \ldots, x_{n}$ and suppose that $H_{p, q}$ satisfies $w=1$. Note that, as a consequence of the identities in (8), we have that commutators commute: indeed, those identities imply that every commutator is a $q$ th power. It follows that, using the identities in (8), $w$ can be reduced to a word of the form $x_{1}^{u_{1}} x_{2}^{u_{2}} \cdots x_{r}^{u_{r}} c_{1}^{u_{1}} \cdots c_{t}^{v_{t}}$ where the $c_{i}$ are distinct commutators of the form $\left[x_{i_{1}}, x_{i_{2}}, \ldots, x_{i_{s}}\right]$. Since $H_{p, q}$ satisfies $w=1$, we immediately deduce that $p q \mid u_{i}(i=1, \ldots, r)$ and so $H_{p, q}$ satisfies $w^{\prime}=1$ where $w^{\prime}=c_{1}^{v_{1}} \cdots c_{t}^{v_{t}}$. Using the last three identities in (8), we may assume that for any $c_{j}=\left[x_{i_{1}}, x_{i_{2}}, \ldots, x_{i_{s}}\right]$ in $w^{\prime}$, the number of occurrences of any variable in $c_{j}$ is less than $q$. Substituting $(1,0)$ for $x_{i_{0}}$ and $\left(0,-b_{i}\right)$ for $x_{i}\left(i \neq i_{0}\right)$, the value assumed by $w^{\prime}$ is of the form $\left(P\left(\omega^{b_{1}}-1, \ldots, \omega^{b_{t_{0}-1}}-1, \omega^{b_{t_{0}+1}}-1, \ldots, \omega^{b_{r}}-1\right), 0\right)$, where $P\left(X_{1}, \ldots, X_{i_{0}-1}, X_{i_{0}+1}, \ldots, X_{r}\right)$ is a polynomial of degree less than $q$ on each variable. Since that value is 0 for any choice of $b_{i} \in \mathbf{Z}_{q}\left(i \neq i_{0}\right)$, it follows that $P$ is identically zero in $F\left[X_{1}, \ldots, X_{i_{0}-1}, X_{i_{0}+1}, \ldots, X_{r}\right]$ and 
thus, the exponents of the commutators $c_{j}=\left[x_{i_{1}}, x_{i_{2}}, \ldots, x_{i_{r}}\right]$ in $w^{\prime}$ for which $i_{0}=i_{1}$ or $i_{0}=i_{2}$, but $i_{0} \neq i_{3}, \ldots, i_{r}$, are multiples of $p$. Hence, we may cancel all these $c_{j}$ for each value of $i_{0}$, yielding a word $w^{\prime \prime}$.

At this point, we may assume that we have an identity $w^{\prime \prime}=1$ which holds in $H_{p, q}$, where $w^{\prime \prime}$ is a product of commutators of the form $\left[x_{i_{1}}, x_{i_{2}}, x_{i_{1}}, x_{i_{2}}, x_{i_{3}}, \ldots, x_{i_{r}}\right]$ with $i_{1}<i_{2}$. Now, if we substitute $\left(\omega^{-a_{1}},-a_{1}\right)$ for $x_{1}$ and $\left(\omega^{-a_{2}} \lambda,-a_{2}\right)$ for $x_{2}$ we obtain

$$
\left[x_{1}, x_{2}\right]=\left(\left(\omega^{a_{2}}-1\right)-\left(\omega^{a_{1}}-1\right) \lambda, 0\right)=(\alpha, 0) .
$$

Hence, any such substitution together with the assignment of the value $\left(0,-a_{i}\right)$ for $x_{i}(i>2)$ gives the value $\left(\alpha P\left(\omega^{a_{i}}-1, \ldots, \omega^{a_{r}}-1\right), 0\right)$ for $w^{\prime \prime}$ where $P\left(X_{1}, \ldots, X_{r}\right)$ is a polynomial of degree less than $q$ on each variable and independent of $\lambda$. With two appropriate choices for $\lambda$ (such as $\lambda=1$ and $\lambda=\omega)$, and recalling that $w^{\prime \prime}=1$ holds in $H_{p, q}$, we deduce that $P\left(\omega^{a_{i}}-1, \ldots, \omega^{a_{r}}-1\right)=0$ for all choices of $a_{1}, \ldots, a_{r} \in \mathbf{Z}_{q}$. Because of the degree restrictions on $P$, it follows that $P$ is identically zero in $F\left[X_{1}, \ldots, X_{r}\right]$. Hence, the exponents of the commutators $\left[x_{1}, x_{2}, \ldots\right]$ in $w^{\prime \prime}$ are all multiples of $p$, and so we can cancel these commutators using the identity $[x, y]^{p}=1$. Since this argument can be repeated for any pair of variables, we conclude that $w^{\prime \prime}=1$ is a consequence of the identities in (8).

This shows that any identity which holds in $H_{p, q}$ follows from the identities in (8) and completes the proof of the proposition.

THEOREM 4.5. The minimal non-commutative pseudovarieties of groups are $\underline{V}\left(G_{p}\right)$ and $\underline{V}\left(H_{p, q}\right)$ with $p$, q distinct primes. These pseudovarieties are all distinct and every non-commutative pseudovariety of groups contains one of them.

Proof. Suppose $\underline{W}$ is a non-commutative pseudovariety of groups. Let $G \in \underline{W}$ be a non-abelian group of minimal order. Then $G$ is one-step non-commutative and so $G$ is isomorphic to one of the groups listed in Theorem 4.1. By Lemma 4.2 and 4.3, together with the remark that the quaternion group generates the pseudovariety in (7), we then have that $\underline{W}$ contains one of the pseudovarieties $\underline{V}\left(G_{p}\right)$ or $\underline{V}\left(H_{p, q}\right)$.

Finally, using (2), (4) and (6)-(8), one can easily check that the particular pseudovarieties in the statement of the theorem are all distinct.

CoRollary 4.6. $G_{p}$ (resp. $\left.H_{p, q}\right)$ is a generator of $\underline{V}\left(G_{p}\right)$ (resp. $\left.\underline{V}\left(H_{p, q}\right)\right)$ of minimum size. 
5. Minimal non-commutative and minimal non-strongly left permutative pseudovarieties of semigroups. We start by introducing some semigroups which will be useful in the sequel.

\section{DEFINITION 5.1.}

$$
\begin{aligned}
N & =\left\langle a, b ; a^{2}=b^{2}=b a=0\right\rangle, \\
T & =\left\langle e, a ; e=e^{2}, a=e a, a e=0\right\rangle, \\
B(m, n) & =\{1, \ldots, m\} \times\{1, \ldots, n\} \text { under }(i, j)(k, l)=(i, l) .
\end{aligned}
$$

Also, recall the semigroup $D$ of Example 3.11. Note that $D$ embeds both $T$ and $T^{r}$. Further, the following equalities can be verified directly, or else encountered in the literature (cf. Edmunds $[4,5])$ :

$$
\begin{aligned}
& \underline{V}(N)=\llbracket x y z=0=x^{2} \rrbracket \\
& \underline{V}(B(1,2))=\llbracket x y=y \rrbracket \\
& \underline{V}(T)=\llbracket x^{2} y=x y, x y^{2}=y x^{2} \rrbracket \\
& \underline{V}\left(N^{1}\right)=\llbracket x^{2}=x^{3}, x^{2} y=x y x=y x^{2} \rrbracket \\
& \underline{V}\left(B(1,2)^{1}\right)=\llbracket x=x^{2}, x y=y x y \rrbracket .
\end{aligned}
$$

Moreover, the indicated semigroups are generators of minimum size of the pseudovarieties they generate.

The following result summarizes Lemmas 2.2-2.6 of Margolis and Pin [10].

Proposition 5.2. (i) Let $\underline{V}$ be â pseudovariety of monoids such that all groups in $\underline{V}$ are abelian and $N^{1}, B(1,2)^{1}, B(2,1)^{1}$ do not lie in $\underline{V}$. Let $M \in \underline{V}$. Then $\mathscr{H}=\mathscr{J}$ is a congruence on $M$ and, if $a, b \in M$ and $a b \neq b a$, then $a b \not{p} b a$ in the submonoid of $M$ generated by $\{a, b\}$.

(ii) Part (i) remains valid if we replace the word "monoid" by "semigroup" and the monoids $N^{1}, B(1,2)^{1}, B(2,1)^{1}$ by the semigroups $N, B(1,2)$, $B(2,1)$.

Here, we present an alternate way of concluding the proof of the characterization of minimal non-commutative pseudovarieties of monoids given in [10], thus avoiding certain language theoretic arguments.

THEOREM 5.3. The minimal non-commutative pseudovarieties of monoids are the ones of Theorem 4.5 together with $\underline{V}\left(N^{1}\right), \underline{V}\left(B(1,2)^{1}\right)$ and $\underline{V}\left(B(2,1)^{1}\right)$. These pseudovarieties are all distinct and every non-commutative pseudovariety of monoids contains one of them. 
Proof. In view of Theorem 4.5 and the defining identities for each of the given pseudovarieties, it suffices to show that, if a pseudovariety $\underline{W}$ does not contain any of the pseudovarieties in our list, then $\underline{W}$ is commutative.

Suppose $M \in \underline{W}, a, b \in M$ and $a b \neq b a$. We may assume that $\{a, b\}$ generates $M$ and, by Proposition 5.2(i), that $M$ is $\mathscr{J}$-trivial. We show that then necessarily $N^{1} \in \underline{W}$ and thus obtain a contradiction.

If $a b, b a \in E(M)$, then $a b a b=a b \mathscr{R} a b a \mathscr{L} b a=b a b a$, and so $a b=$ $a b a=b a$. Suppose then $a b$ is not an idempotent. If $b a$ is an idempotent, then $b a<_{\mathscr{J}} a b$. Since $a b \neq b a$ and $M$ is $\mathscr{J}$-trivial, either $a b{ }_{\mathscr{J}} b a$ or $b a \nless g a b$. Thus, we may assume that $a b \nless{ }_{\mathscr{J}} b a$.

Let $I=\{x \in M: a b \nless g x\}$, an ideal of $M$. Let $M^{\prime}=M / I$ and $M^{\prime \prime}=M^{\prime} \times M^{\prime} \times M^{\prime}$. Let $I^{\prime \prime}$ be the ideal of $M^{\prime \prime}$ consisting of all triples with at least one zero component. Finally, let $M^{\prime \prime \prime}=M^{\prime \prime} / I^{\prime \prime}$ and let $x=(a, 1, b), y=(b, a b, 1)$. Then $x^{2}=y^{2}=y x=0$ but $1, x, y, x y$, and 0 are all distinct in $M^{\prime \prime \prime}$. Hence, $N^{1} \simeq\{1, x, y, x y, 0\} \subseteq M^{\prime \prime \prime}$ and so $N^{1} \in \underline{W}$.

The next result corrects Theorem 2.8 [10].

THEOREM 5.4. The minimal non-commutative pseudovarieties of semigroups are the ones of Theorem 4.5 together with $\underline{V}(N), \underline{V}(B(1,2))$, $\underline{V}(B(2,1)), \underline{V}(T)$, and $\underline{V}\left(T^{r}\right)$. These pseudovarieties are all distinct and every non-commutative pseudovariety of semigroups contains one of them.

Proof. As in the proof of Theorem 5.3, we only need to consider a pseudovariety $\underline{W}$ (of semigroups) which does not contain any of the pseudovarieties in the given list and show that $\underline{W}$ must be commutative.

Suppose $S \in \underline{W}, a, b \in S$, and $a b \neq b a$. Again, we assume that $S$ is generated by $\{a, b\}$ and, in view of Proposition 5.2(ii), that $S$ is $\mathscr{J}$-trivial. And, just as in the monoid case, we may here assume that $a b$ is not an idempotent, $a b{ }_{\mathscr{J}} b a$, and that the ideal $\left\{x \in S: a b{ }_{\mathscr{J}} x\right\}$ of $S$ is reduced to zero.

Let $e=a^{\omega}, f=b^{\omega}$ (cf. §3). If $a^{n}=a b$, then $a^{n+1}=a b a=0$, so that $e=0$. Similarly, if $b^{m}=a b$, then $f=0$. If $e \neq 0$, then $a b=x e y$ for some $x, y \in S$; since $b a=0$, it follows that $x=e a^{i}$ for some $i>0$, and so $e a b=a b$ and $a b=e b$ (as then $a b \mathscr{L} e b$ ). Similarly, if $f \neq 0$, then $a b f=a b$ $=a f$.

There are several cases to be considered.

(i) $e \neq 0$ and $f \neq 0$. Then, by the above $a b=e f$. Thus, $\{e, f, e f, 0\}$ $\simeq D$, so that $T, T^{r} \in \underline{W}$.

(ii) $e \neq 0$ and $f=0$. Then $\{e, e b, 0\} \simeq T$ and so $T \in \underline{W}$.

(iii) $e=0$ and $f \neq 0$. Then $\{f, a f, 0\} \simeq T^{r}$ and so $T^{r} \in \underline{W}$. 
(iv) $e=0$ and $f=0$. Consider the subsemigroup $S^{\prime}$ of $S \times S \times S$ generated by the elements $\alpha=(a, a, a b)$ and $\beta=(b, a b, b)$. Then $\alpha \beta=$ $(a b, 0,0) \neq 0=(0,0,0)=\beta \alpha$. Let $I^{\prime}=\left\{x \in S^{\prime}: \alpha \beta \nless{ }_{\mathscr{J}} x\right\}$, an ideal of $S^{\prime}$. Note that $\alpha^{2}, \beta^{2}, \beta \alpha \in I^{\prime}$, while $\alpha, \beta$, and $\alpha \beta$ are distinct elements of $S^{\prime} \backslash I^{\prime}$. Hence, $S^{\prime} / I^{\prime} \simeq N$ and $N \in \underline{W}$.

In all cases we reach a contradiction, whence the proof is complete.

LeMma 5.5. Let $\underline{W}$ be a pseudovariety of semigroups and $S \in \underline{W}$. Let $e \in E(S)$ and $s \in S$.

(i) If se $\mathscr{J}$ ese, then $T^{r} \in \underline{W}$.

(ii) If se $\mathscr{J}$ ese, then either se $=$ ese or $B(2,1) \in \underline{W}$.

Proof. (i) Suppose that se $\mathscr{Z}$ ese. Then ese $<$ se. Consider the Rees quotient $S^{\prime}$ of the subsemigroup of $S$ generated by $\{e, s\}$ by its ideal $\{x$ : se $\nless g x\}$. Then $\{e$, se, 0$\} \simeq T^{r}$ and so $T^{r} \in \underline{W}$.

(ii) Suppose that se $\mathscr{J}$ ese. Then $s e \mathscr{L}$ ese, say se=tese for some $t \in S$. Let $f=(t e)^{\omega}$. Then $s e=f s e$ and $f e=f$. Hence, $f e f=f$ and so ef $\in E(S)$ and ef $\mathscr{L} f$. Thus, either ef $\neq f$ and $B(2,1) \simeq\{e f, f\} \in \underline{W}$, or ef $=f=f e$. In the latter case,

$$
s e=f s e=e f s e=e s e .
$$

THEOREM 5.6. A pseudovariety of semigroups is not strongly permutative if and only if it contains one of the pseudovarieties in Theorem 4.5 or one of $\underline{V}\left(N^{1}\right), \underline{V}(B(1,2)), \underline{V}(B(2,1)), \underline{V}(T)$, or $\underline{V}\left(T^{r}\right)$. Moreover, these pseudovarieties are distinct minimal non-strongly permutative.

Proof. It remains to show the only if part of the statement. Suppose $\underline{W}$ is a pseudovariety of semigroups which does not contain any of the pseudovarieties listed in the theorem. By Theorems 3.3(iv) and 5.3, it suffices to show that if $S \in \underline{W}, s \in S$ and $e \in E(S)$, then $e s=s e$. But, by Lemma 5.5 and its left-right dual, we have $e s=e s e=s e$, as desired.

THEOREM 5.7. A pseudovariety of semigroups is not strongly left permutative if and only if it contains one of the pseudovarieties in Theorem 4.5 or one of $\underline{V}\left(N^{1}\right), \underline{V}\left(B(1,2)^{1}\right), \underline{V}(B(2,1))$, or $\underline{V}\left(T^{r}\right)$. Moreover, these pseudovarieties are distinct minimal non-strongly left permutative.

Proof. The result now follows from Theorems 3.5(iv) and 5.3 along with Lemma 5.5.

REMARK 5.8. Theorem 5.6 may also be deduced from Theorem 5.7 and its left-right dual. 


\section{REFERENCES}

[1] J. Almeida, On power varieties of semigroups, manuscript, Simon Fraser University.

[2] C. J. Ash, Pseudovarieties, generalized varieties and similarly described classes, to appear in J. Algebra.

[3] A. H. Clifford and G. B. Preston, The Algebraic Theory of Semigroups, Amer. Math. Soc., Math. Surveys, No. 7, Providence, R.I., 1961, 1967.

[4] C. C. Edmunds, On certain finitely based varieties of semigroups, Semigroup Forum, 15 (1977), 21-39.

[5] _ Varieties generated by semigroups of order four, Semigroup Forum, 21 (1980), 67-81.

[6] S. Eilenberg, Automata, Languages and Machines, Vol. B, Academic Press, New York, 1976.

[7] P. M. Higgins, Epimorphisms, permutation identities and finite semigroups, manuscript, Monash University.

[8] - The permutation identities which ensure that a semigroup variety is finitely based, manuscript, Monash University.

[9] G. Lallement, Semigroups and Combinatorial Applications, Wiley-Interscience, New York, 1979.

[10] S. W. Margolis and J. E. Pin, Minimal non-commutative varieties and power varieties, Pacific J. Math., 111 (1984), 125-135.

[11] H. Neumann, Varieties of Groups, Springer-Verlag, New York, 1967.

[12] P. Perkins, Bases for equational theories of semigroups, J. Algebra, 11 (1968), 298-314.

[13] G. Pollák, On the consequences of permutation identities, Acta Sci. Math., 34 (1973), 323-333.

[14] _ On hereditarily finitely based varieties of semigroups, Acta Sci. Math. (Szeged) 37, No. 3-4 (1975), 339-348.

[15] _ On identities which define hereditarily finitely based varieties of semigroups, Coll. Math. Soc. János Bolyai 20, Algebraic Theory of Semigroups, Szeged, Hungary, 1976, 447-452.

[16] M. S. Putcha and A. Yaqub, Semigroups satisfying permutation identities, Semigroup Forum, 3 (1971), 68-73.

[17] L. Rédei, Algebra, Vol. 1 (English translation), Pergamon Press, Oxford, 1967.

[18] J. Reiterman, The Birkhoff theorem for finite algebras, Algebra Universalis, 14 (1982), $1-10$.

[19] M. Yamada, Inversive semigroups III, Nihon Gakushiin, Proc., 41 (1965), 221-224.

[20] _ Regular semigroups whose idempotents satisfy permutation identities, Pacific J. Math., 21, No. 2 (1967),371-392.

Received April 10, 1984. This work was supported, in part, by NSERC Grant A4044.

Centro de MATEMÁtica

UNIVERSIDADE DO MINHO

4700 Braga, Portugal 


\title{
PACIFIC JOURNAL OF MATHEMATICS EDITORS
}

V. S. VARADARAJAN (Managing Editor)
University of California
Los Angeles, CA 90024
HEBERT ClEMENS
University of Utah
Salt Lake City, UT 84112
CHARLES R. DEPRIMA
California Institute of Technology
Pasadena, CA 91125

R. FINN

Stanford University

Stanford, CA 94305

HERMANN FLASCHKA

University of Arizona

Tucson, AZ 85721

Ramesh A. Gangolli

University of Washington

Seattle, WA 98195

ROBION KIRBY

University of California

Berkeley, CA 94720
C. C. MOORE

University of California

Berkeley, CA 94720

H. SAMELSON

Stanford University

Stanford, CA 94305

HaRold Stark

University of California, San Diego

La Jolla, CA 92093

\section{ASSOCIATE EDITORS}

\author{
R. ARENS \\ E. F. BECKENBACH \\ B. H. NeumanN \\ F. Wolf \\ K. YoSHIDA \\ (1906-1982)
}

\section{SUPPORTING INSTITUTIONS}

UNIVERSITY OF ARIZONA
UNIVERSITY OF BRITISH COLUMBIA
CALIFORNIA INSTITUTE OF TECHNOLOGY
UNIVERSITY OF CALIFORNIA
MONTANA STATE UNIVERSITY
UNIVERSITY OF NEVADA, RENO
NEW MEXICO STATE UNIVERSITY
OREGON STATE UNIVERSITY

UNIVERSITY OF OREGON

UNIVERSITY OF SOUTHERN CALIFORNIA

STANFORD UNIVERSITY

UNIVERSITY OF HAWAII

UNIVERSITY OF TOKYO

UNIVERSITY OF UTAH

WASHINGTON STATE UNIVERSITY UNIVERSITY OF WASHINGTON

The Supporting Institutions listed above contribute to the cost of publication of this Journal, but they are not owners or publishers and have no responsibility for its content or policies.

Mathematical papers intended for publication in the Pacific Journal of Mathematics should be in typed form or offset-reproduced (not dittoed), double spaced with large margins. Please do not use built up fractions in the text of the manuscript. However, you may use them in the displayed equations. Underline Greek letters in red, German in green, and script in blue. The first paragraph must be capable of being used separately as a synopsis of the entire paper. In particular it should contain no bibliographic references. Please propose a heading for the odd numbered pages of less than 35 characters. Manuscripts, in triplicate, may be sent to any one of the editors. Please classify according to the scheme of Math. Reviews, Index to Vol. 39. Supply name and address of author to whom proofs should be sent. All other communications should be addressed to the managing editor, or Elaine Barth, University of California, Los Angeles, California 90024.

There are page-charges associated with articles appearing in the Pacific Journal of Mathematics. These charges are expected to be paid by the author's University, Government Agency or Company. If the author or authors do not have access to such Institutional support these charges are waived. Single authors will receive 50 free reprints; joint authors will receive a total of 100 free reprints. Additional copies may be obtained at cost in multiples of 50 .

The Pacific Journal of Mathematics is issued monthly as of January 1966. Regular subscription rate: $\$ 190.00$ a year (5 Vols., 10 issues). Special rate: $\$ 95.00$ a year to individual members of supporting institutions.

Subscriptions, orders for numbers issued in the last three calendar years, and changes of address should be sent to Pacific Journal of Mathematics, P.O. Box 969, Carmel Valley, CA 93924, U.S.A. Old back numbers obtainable from Kraus Periodicals Co., Route 100, Millwood, NY 10546.

The Pacific Journal of Mathematics at P.O. Box 969, Carmel Valley, CA 93924 (ISSN 0030-8730) publishes 5 volumes per year. Application to mail at Second-class postage rates is pending at Carmel Valley, California, and additional mailing offices. Postmaster: Send address changes to Pacific Journal of Mathematics, P.O. Box 969, Carmel Valley, CA 93924.

\section{PUBLISHED BY PACIFIC JOURNAL OF MATHEMATICS, A NON-PROFIT CORPORATION}

Copyright $\subset 1986$ by Pacific Journal of Mathematics 


\section{Pacific Journal of Mathematics}

\section{Vol. 121, No. 2 December, 1986}

Jorge Almeida, Minimal nonpermutative pseudovarieties of semigroups.

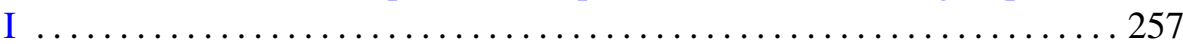

Jorge Almeida, Minimal nonpermutative pseudovarieties of semigroups.

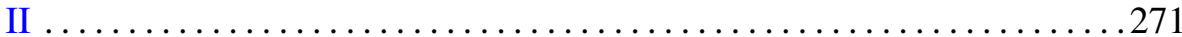

Carlos Andradas Heranz and José Manuel Gamboa Mutuberría, On

projections of real algebraic varieties $\ldots \ldots \ldots \ldots \ldots \ldots \ldots \ldots \ldots 281$

Zeev Ditzian, Inverse theorems for multidimensional Bernstein operators . . 293

M. Furi and Maria Patrizia Pera, A continuation principle for forced oscillations on differentiable manifolds $\ldots \ldots \ldots \ldots \ldots \ldots \ldots \ldots \ldots . \ldots . \ldots . \ldots 321$

James J. Hebda, The collars of a Riemannian manifold and stable

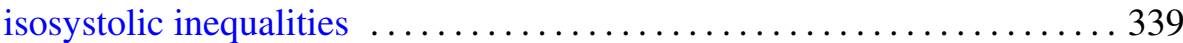

Henryk Hecht and Dragan Miličić, Character identities and asymptotic behavior of matrix coefficients of discrete series ................ 357

Piotr Jakóbczak, The boundary regularity of the solution of the $\bar{\partial}$-equation in the product of strictly pseudoconvex domains $\ldots \ldots \ldots \ldots \ldots \ldots . \ldots 371$

Krzysztof Jarosz, Isometries between injective tensor products of Banach

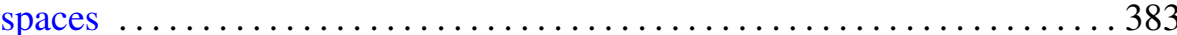

Hans Keller, On valued, complete fields and their automorphisms ........ 397

David Masser and Peter Man-Kit Shiu, On sparsely totient numbers . . . . 407

Tze-Beng Ng, Vector bundles over $(8 k+3)$-dimensional manifolds

Thomas Joseph Ransford, The spectrum of an interpolated operator and analytic multivalued functions

Akihito Uchiyama, On the radial maximal function of distributions 467 Jang-Mei Gloria Wu, On singularity of harmonic measure in space 